\title{
Antibacterial and Antioxidant Activity of Solanum nigrum Stem and Leaves
}

\author{
POOJA UPADHYAY*, SHABINA ARA and POONAM PRAKASH
}

Department of Chemistry, School of Basic Science, Sam Higginbottom Institute of Agriculture, Technology \& Sciences, (Deemed University) Allahabad- 211007, India

poojaravit2011@gmail.com

Received 15 July 2015 / Accepted 24 July 2015

\begin{abstract}
The antioxidant and antibacterial activity of stem and leaves extracts of Solanum nigrum, which is an important ingredient in traditional Indian medicines was determined in this work. It was tested for antioxidant activity using 2, 2- diphenyl- 1- picrylhydrazyl (DPPH) and reducing power assay method. The antibacterial activity was detected by agar well diffusion method against Bacillus subtilis, Escherichia coli and Pseudomonas aeruginosa. The zones of inhibitions obtained were recorded and analyzed against standard control of Ampicillin.
\end{abstract}

Keywords: DPPH, Reducing power assay, Ascorbic acid, Agar diffusion

\section{Introduction}

For thousands of year's natural products have played a very important role in health care and prevention of diseases. Forty- seven percent of the anticancer drugs in the market come from natural products or natural product mimics ${ }^{1}$. Important drugs such as taxol, camptothecin, morphine and quinine have been isolated from plant sources. The plant possesses various chemotherapeutic, bacteriostatic and antimicrobial agents ${ }^{2}$. Plants generally produce many secondary metabolites which constitute an important source of microbicides, pesticides and many pharmaceutical drugs. Several types of polyphenols (Phenolic acid, Hydrolysable tannins and Flavonoids) show anticarcinogenic and antimutagenic effects ${ }^{3}$. Plant derived natural products have received considerable attention in recent years due to diverse pharmacological properties, including antioxidant and antitumor activity ${ }^{4}$.

Solanum nigrum L and S. Myria canthus, both are important aspects of medicinal plant resources for treatment of primary health care. Solanum nigrum L commonly known as Black nightshade is a dicot weed in the Solanaceae family. The herb is antiseptic, antidysentric antidiuretic used in the treatment of cardiac, skin disease, psoriasis, herpvirus and inflammation of kidney ${ }^{5}$. 


\section{Experimental}

Plants were collected from campus of SHIATS and identified from the Department of agronomy, SHIATS, Allahabad.

\section{Preparation of extract}

The dried plant material (stem and leaves) of Solanum nigrum was made to fine powder using, homogenizer. The dried powder was extracted separately with continuous shaking for $24 \mathrm{~h}$ using different solvents. The extracts are filtered through filter paper to remove all unextractable matters.

\section{Microorganisms}

The antibacterial activity was tested against the following 3 selected strains: Escherichia coli, Pseudomonas aeruginosa and Bacillus subtilis. The selected bacterial strains were obtained from Microbiology\& Fermentation technology laboratory, SHIATS, Allahabad.

\section{Evaluation of antibacterial activity}

Agar well diffusion method

Agar well diffusion method elucidated by Ahmad et al. ${ }^{6}$ was followed.

\section{Evaluation of antioxidant activity}

\section{Scavenging activity on DPPH radical}

The DPPH radical scavenging assays elucidated by Chan et al. ${ }^{7}$ was followed.

\section{Reducing power assay}

Antioxidant activity by reducing power assay developed by Yen and $\operatorname{Duh}^{8}$ was as followed.

\section{Results and Discussion}

The result of antibacterial activity is given in Table 1, 2, 3 and 4. From the tables it is clear that the chloroform: methanol extract of stem of Solanum nigrum was highly active against Bacillus subtilis and the acetone extract against Pseudomonas aeruginosa at $2.0 \mathrm{mg} / \mathrm{mL}$. The chloroform:methanol extracts of leaf of Solanum nigrum showed maximum inhibitory activity against ${ }^{9}$ Pseudomonas aeruginosa and the acetone extract against Pseudomonas aeruginosa at $2.0 \mathrm{mg} / \mathrm{mL}$. According to the Table 5, $2.0 \mu \mathrm{g} / \mathrm{mL}$ concentration of Ampicillin showed maximum radius of zone of inhibition of $22.0 \mathrm{~mm}$ against $E$. coli followed by 19.0 $\mathrm{mm}$ against $B$. subtilis and $23.0 \mathrm{~mm}$ P. aureginosa ${ }^{10}$.

Table 1. Antibacterial activity of Solanum nigrum chloroform: methanol (3:2) leaf extract by agar well diffusion method

\begin{tabular}{cccc}
\hline \multirow{2}{*}{$\begin{array}{c}\text { Concentration } \\
\mathrm{mg} / \mathrm{mL}\end{array}$} & \multicolumn{3}{c}{ Mean radius of inhibition zones of bacteria, mm } \\
\cline { 2 - 4 } & E. coli & B. subtilis & P. aeruginosa \\
\hline 0.5 & 7.0 & 6.0 & 8.0 \\
1.0 & 7.0 & 7.0 & 8.0 \\
1.5 & 9.0 & 8.0 & 11.0 \\
2.0 & 9.0 & 10.0 & 11.0 \\
\hline
\end{tabular}


Table 2. Antibacterial activity of Solanum nigrum acetone leaf extract by agar well diffusion

\begin{tabular}{cccc}
\hline Concentration & \multicolumn{3}{c}{ Mean radius of inhibition zones of bacteria, $\mathrm{mm}$} \\
\cline { 2 - 4 } $\mathrm{mg} / \mathrm{mL}$ & E. coli & B. subtilis & P. aeruginosa \\
\hline 0.5 & 6.0 & 7.0 & 8.0 \\
1.0 & 6.0 & 7.0 & 9.0 \\
1.5 & 8.0 & 8.0 & 9.0 \\
2.0 & 8.0 & 9.0 & 10.0 \\
\hline
\end{tabular}

Table 3. Antibacterial activityof Solanum nigrum chloroform: methanol (3:2) stem extract by agar well diffusion

\begin{tabular}{cccc}
\hline Concentration & \multicolumn{3}{c}{ Mean radius of inhibition zones of bacteria, $\mathrm{mm}$} \\
\cline { 2 - 4 } $\mathrm{mg} / \mathrm{mL}$ & E. coli & B. subtilis & P. aeruginosa \\
\hline 0.5 & 7.0 & 6.0 & 6.0 \\
1.0 & 9.0 & 6.0 & 7.0 \\
1.5 & 14.0 & 12.0 & 10.0 \\
2.0 & 17.0 & 18.0 & 11.0 \\
\hline
\end{tabular}

Table 4. Antibacterial activity of Solanum nigrum acetone stem extract by agar well diffusion

\begin{tabular}{cccc}
\hline Concentration & \multicolumn{3}{c}{ Mean radius of inhibition zones of bacteria, mm } \\
\cline { 2 - 4 } $\mathrm{mg} / \mathrm{mL}$ & E. coli & B. subtilis & P. aeruginosa \\
\hline 0.5 & 6.0 & 6.0 & 6.0 \\
1.0 & 7.0 & 9.0 & 9.0 \\
1.5 & 8.0 & 9.0 & 10.0 \\
2.0 & 10.0 & 10.0 & 11.0 \\
\hline
\end{tabular}

Table 5. Antibacterial activity of standard Ampicillin by agar well diffusion

\begin{tabular}{cccc}
\hline Concentration & \multicolumn{3}{c}{ Mean radius of inhibition zones of bacteria, mm } \\
\cline { 2 - 4 } $\mathrm{mg} / \mathrm{mL}$ & E. coli & B. subtilis & P. aeruginosa \\
\hline 0.5 & 15.0 & 15.0 & 17.0 \\
1.0 & 16.0 & 15.0 & 17.0 \\
1.5 & 19.0 & 17.0 & 18.0 \\
2.0 & 22.0 & 19.0 & 23.0 \\
\hline
\end{tabular}

Table 6. DPPH free radical scavenging assay (\%) ofSolanum nigrum leaf

\begin{tabular}{ccccc}
\hline \multirow{2}{*}{ Extracts } & \multicolumn{4}{c}{ \% inhibition at different concentration, $\mu \mathrm{g} / \mathrm{mL}$} \\
\cline { 2 - 5 } & $200, \mu \mathrm{g} / \mathrm{mL}$ & $400, \mu \mathrm{g} / \mathrm{mL}$ & $600, \mu \mathrm{g} / \mathrm{mL}$ & $800, \mu \mathrm{g} / \mathrm{mL}$ \\
\hline Ether & $0.083 \pm 0.0049$ & $0.076 \pm 0.0078$ & $0.069 \pm 0.0015$ & $0.063 \pm 0.0025$ \\
$\%$ & 45.75 & 50.33 & 54.90 & 58.82 \\
Ethyl acetate & $0.148 \pm 0.0050$ & $0.139 \pm 0.0055$ & $0.128 \pm 0.0035$ & $0.112 \pm 0.0075$ \\
$\%$ & 48.43 & 51.57 & 55.40 & 60.98 \\
Chloroform: methanol & $0.046 \pm 0.0040$ & $0.041 \pm 0.0061$ & $0.037 \pm 0.0025$ & $0.033 \pm 0.0031$ \\
$(3: 2)$ & 71.78 & 74.85 & 77.30 & 79.75 \\
$\%$ & $0.167 \pm 0.0240$ & $0.156 \pm 0.0300$ & $0.144 \pm 0.0356$ & $0.127 \pm 0.0324$ \\
Acetone & 42.41 & 46.21 & 50.34 & 56.21 \\
$\%$ & $0.033 \pm 0.0021$ & $0.030 \pm 0.0025$ & $0.028 \pm 0.0020$ & $0.024 \pm 0.0032$ \\
Ascorbic acid & 84.29 & 85.71 & 86.67 & 88.57 \\
$\%$ & &
\end{tabular}

Values were expressed as MEAN+S.D. $(n=3)$ 
The result of antioxidant activity by DPPH method showed that the leaf extract of chloroform methanol (3:2) has the highest value of percentage inhibition of DPPH $79.75 \%$ whereas stem extracted by same solvent showed $68.78 \%$ at concentration of 800 $\mu \mathrm{g} / \mathrm{mL}$. In this method leaf extract showed better result than stem extracts of Solanum nigrum $^{11}$.

The result of antioxidant activity by reducing power assay as shown in Table 8 and 9, it is evident that the leaf extract and stem extract of chloroform: methanol (3:2) showed maximum absorbance at $800 \mu \mathrm{g} / \mathrm{mL} 0.362$ and 0.298 respectively. Highest activity of chloroform: methanol extract for leaf and stem is according to the result of Sudhanshu et al. ${ }^{12}$ which studied that methanol extract of Solanum nigrum showed highest antioxidant activity.

Table 7. DPPH free radical scavenging assay (\%) of Solanum nigrum stem

\begin{tabular}{ccccc}
\hline \multirow{2}{*}{ Extracts } & \multicolumn{4}{c}{ \% Inhibition at different concentration, $\mu \mathrm{g} / \mathrm{mL}$} \\
\cline { 2 - 5 } & $200, \mu \mathrm{g} / \mathrm{mL}$ & $400, \mu \mathrm{g} / \mathrm{mL}$ & $600, \mu \mathrm{g} / \mathrm{mL}$ & $800, \mu \mathrm{g} / \mathrm{mL}$ \\
\hline Ether & $0.124 \pm 0.0147$ & $0.117 \pm 0.0137$ & $0.097 \pm 0.0121$ & $0.086 \pm 0.0152$ \\
$\%$ & 29.94 & 33.89 & 45.19 & 51.41 \\
Ethyl acetate & $0.134 \pm 0.0032$ & $0.122 \pm 0.0026$ & $0.105 \pm 0.0047$ & $0.086 \pm 0.0074$ \\
$\%$ & 29.47 & 35.79 & 44.74 & 54.74 \\
Chloroform: methanol & $0.087 \pm 0.0020$ & $0.077 \pm 0.0066$ & $0.064 \pm 0.0031$ & $0.059 \pm 0.0036$ \\
$(3: 2)$ & 53.97 & 59.26 & 66.14 & 68.78 \\
$\%$ & $0.214 \pm 0.0107$ & $0.201 \pm 0.0025$ & $0.191 \pm 0.0028$ & $0.186 \pm 0.0010$ \\
Acetone & 25.44 & 29.96 & 33.45 & 35.19 \\
$\%$ & $0.033 \pm 0.0021$ & $0.030 \pm 0.0025$ & $0.028 \pm 0.0020$ & $0.024 \pm 0.0032$ \\
Ascorbic acid & 84.29 & 85.71 & 86.67 & 88.57 \\
$\%$ & & & &
\end{tabular}

Table 8. Reducing power activity of Solanum nigrum leaf

\begin{tabular}{cccccc}
\hline $\begin{array}{c}\text { Concentration } \\
\mu \mathrm{g} / \mathrm{mL}\end{array}$ & $\begin{array}{c}\text { Ascorbic } \\
\text { acid }\end{array}$ & Ether & $\begin{array}{c}\text { Ethyl } \\
\text { acetate }\end{array}$ & $\begin{array}{c}\text { Chloroform } \\
\text { methanol (3:2) }\end{array}$ & Acetone \\
\hline 200 & $0.545 \pm 0.0030$ & $0.262 \pm 0.0010$ & $0.280 \pm 0.0020$ & $0.291 \pm 0.0015$ & $0.211 \pm 0.0010$ \\
400 & $0.604 \pm 0.0074$ & $0.265 \pm 0.0015$ & $0.292 \pm 0.0031$ & $0.318 \pm 0.0122$ & $0.219 \pm 0.0015$ \\
600 & $0.634 \pm 0.0053$ & $0.281 \pm 0.0032$ & $0.295 \pm 0.0045$ & $0.325 \pm 0.0134$ & $0.241 \pm 0.0021$ \\
800 & $0.695 \pm 0.0061$ & $0.291 \pm 0.0066$ & $0.306 \pm 0.0072$ & $0.362 \pm 0.0055$ & $0.252 \pm 0.0025$ \\
\hline \multicolumn{6}{c}{ Values were expressed as MEAN \pm S.D. $(n=3)$}
\end{tabular}

Table 9. Reducing power activity of Solanum nigrum stem

\begin{tabular}{cccccc}
\hline $\begin{array}{c}\text { Concentration } \\
\mu \mathrm{g} / \mathrm{mL}\end{array}$ & $\begin{array}{c}\text { Ascorbic } \\
\text { acid }\end{array}$ & Ether & $\begin{array}{c}\text { Ethyl } \\
\text { acetate }\end{array}$ & $\begin{array}{c}\text { Chloroform: } \\
\text { methanol }(3: 2)\end{array}$ & Acetone \\
\hline 200 & $0.545 \pm 0.0030$ & $0.130 \pm 0.0031$ & $0.180 \pm 0.0064$ & $0.263 \pm 0.0025$ & $0.113 \pm 0.0025$ \\
400 & $0.604 \pm 0.0074$ & $0.140 \pm 0.0064$ & $0.193 \pm 0.0020$ & $0.275 \pm 0.0015$ & $0.139 \pm 0.0026$ \\
600 & $0.634 \pm 0.0053$ & $0.161 \pm 0.0025$ & $0.213 \pm 0.0030$ & $0.289 \pm 0.0010$ & $0.152 \pm 0.0020$ \\
800 & $0.695 \pm 0.0061$ & $0.182 \pm 0.0025$ & $0.218 \pm 0.0025$ & $0.298 \pm 0.0030$ & $0.162 \pm 0.0017$ \\
\hline
\end{tabular}

Values were expressed as MEAN \pm S.D. $(n=3)$ 


\section{Conclusion}

Thus it is concluded that leaf extract of Solanum nigrum can effectively scavenge an assortment of reactive oxygen species or free radicals than stem extract and the antibacterial activity exhibited by extracts of Solanum nigrum leaf and stem material was however less than the standard drugs used.

\section{References}

1. Newman D J and Crag G M, J Nat Prod., 2007, 70(3), 461-477; DOI:10.1021/np068054v

2. Venkatesan D, Karrunakaran C M and Selva S K, Ethnobotanical Leaflets, 2009, 13(1), 1485-1503.

3. Uruguiaga I and Leighton F, Biolog Res., 2000, 33, 159-165.

4. Karthikumar S, Vigneswari K and Jegatheesan K, Sci Res Essay, 2007, 2(4), 101-104.

5. Yogananath N, Buvaneswari S and Muthezhilan R, Global J Biotechnol Biochem., 2012, 7(3), 86-89

6. Ahmad I and Beg A Z, J Ethnopharmacol., 2001, 74(2), 113-123; DOI:10.1016/S0378-8741(00)00335-4

7. $\quad$ Chan E W C, Lim Y Y and Omar M, J Food Chem., 2007, 104(4), 1586-1593; DOI:10.1016/j.foodchem.2007.03.023

8. Yen G C and Duh P D, J Am Oil Chem Soc., 1993, 70(4), 383-386; DOI:10.1007/BF02552711

9. $\quad$ Pandey A, Rajpoot S and Mondal S, Int J Pharma Res Allied Sci., 2012, 1(4), 108120, ISSN 2277-3657.

10. Salar R K and Suchitra, Afr J Microbiol Res., 2009, 3(3), 97-100

11. Jayachitra A and Krithiga N, Int J Med Arom Plants., 2012, 2(3), 495-500.

12. Sudhanshu Rao N, Mittal S and Menghani E, J Adv Pharma Res., 2012, 3(1), 9-13. 